Bull. Austral. Math. Soc.

VOL. 65 (2002) [79-91]

\title{
LINEAR MAPS ON VON NEUMANN ALGEBRAS PRESERVING ZERO PRODUCTS OR TR-RANK
}

\author{
Cui Jianlian and Hou Jinchuan
}

\begin{abstract}
In this paper, we give some characterisations of homomorphisms on von Neumann algebras by linear preservers. We prove that a bounded linear surjective map from a von Neumann algebra onto another is zero-product preserving if and only if it is a homomorphism multiplied by an invertible element in the centre of the image algebra. By introducing the notion of tr-rank of the elements in finite von Neumann algebras, we show that a unital linear map from a linear subspace $\mathcal{M}$ of a finite von Neumann algebra $\mathcal{R}$ into $\mathcal{R}$ can be extended to an algebraic homomorphism from the subalgebra generated by $\mathcal{M}$ into $\mathcal{R}$; and a unital self-adjoint linear map from a finite von Neumann algebra onto itself is completely tr-rank preserving if and only if it is a spatial *-automorphism.
\end{abstract}

\section{INTRODUCTION}

By a linear preserver we mean a linear map from an algebra into another which, roughly speaking, preserves certain properties of some elements in the algebra. Linear preserver problems concern the characterisation of such maps. Over the past decades a lot of work has been done on linear preserver problems on matrix algebras (see, for example, the survey paper [11]). Recently, interest in similar questions on operator algebras over infinite dimensional spaces has also been growing (for example, [1, $3,4,5,7,8,9,11,12,13,14,15,16]$ and the references therein). In this paper, we consider the question how to describe a linear map from a von Neumann algebra into another which preserves zero products or tr-rank, and then get some characterisations of homomorphisms on von Neumann algebras.

Let $\mathcal{A}$ and $\mathcal{B}$ be two algebras. We say that a linear map $\Phi: \mathcal{A} \rightarrow \mathcal{B}$ preserves zero products if $A B=0$ implies that $\Phi(A) \Phi(B)=0$ for any $A, B \in \mathcal{A} ; \Phi$ preserves zero products in both directions if $A B=0$ if and only if $\Phi(A) \Phi(B)=0$ for any $A, B \in \mathcal{A}$. Semrl [15] proved that a unital linear map from $\mathcal{B}(X)$ onto itself is an automorphism if and only if it preserves zero products in both directions, where $\mathcal{B}(X)$ is the Banach algebra of all bounded linear operators on Banach space $X$. In [9], Hou and Gao gave a characterisation of the additive maps preserving zero products on $\mathcal{B}(H)$, where $H$ is

Received 14th May, 2001

This work is supported by NNSFC and PNSFS.

Copyright Clearance Centre, Inc. Serial-fee code: 0004-9727/02 \$A2.00+0.00. 
a Hilbert space. In a recent paper [5], we described all linear or additive maps on nest algebras which preserve zero products. All these results mentioned above are obtained by showing that the maps involved are rank-one preserving, but this method is not valid for those acting on general von Neumann algebras. In the present paper, one of our purposes is to generalise the result of Semrl [15] to general von Neumann algebras and also omit the assumptions "in both directions" and "unitality", under the assumption of boundedness. In Section 2, we prove that a bounded surjective linear map from a von Neumann algebra onto a factor Banach algebra preserves zero products if and only if it is a homomorphism multiplied by a nonzero scalar (Theorem 2.1). We also show that a bounded surjective linear map from a von Neumann algebra $\mathcal{A}$ onto another $\mathcal{B}$ preserves zero products if and only if it is a homomorphism multiplied by an invertible element in the centre of $\mathcal{B}$ (Theorem 2.3).

One of the most important linear preserver problems concerns the rank. This is because many preserver problems may be reduced to the problem of rank preservers. Therefore it is not surprising that there are many papers on linear maps preserving rank (see, for example, $[1,3,4,7,8,11,12,13,14,15,16]$ and the references therein). However, the notion of finite-rank is meaningless in general von Neumann algebras. In Section 3, we introduce the notion of tr-rank of the matrices over a finite von Neumann algebra (see Definition 3.1) and give some properties of tr-rank (Proposition 3.2). It is seen that tr-rank is a suitable generalisation of the rank of matrices. We prove that a unital linear map which is completely tr-rank nonincreasing from a linear subspace $\mathcal{M}$ of a finite von Neumann algebra $\mathcal{R}$ into $\mathcal{R}$ can be extended to a completely tr-rank nonincreasing algebraic homomorphism from the subalgebra generated by $\mathcal{M}$ into $\mathcal{R}$ (Theorem 3.3); if a unital linear map from $\mathcal{R}$ into itself is completely tr-rank nonincreasing, then it is a homomorphism (Corollary 3.4); and a unital self-adjoint linear map from $\mathcal{R}$ onto itself is completely tr-rank preserving if and only if it is a spatial *-automorphism (Theorem 3.6).

\section{LINEAR MAPS PRESERVING ZERO-PRODUCT}

In this section, we discuss the linear maps preserving zero products on von Neumann algebras. In fact, the results of this section are also true if the domain space $\mathcal{A}$ is a $C^{*}$-algebra of real rank zero. Recall that a $C^{*}$-algebra $\mathcal{A}$ is of real rank zero if the set of all real linear combinations of orthogonal self-adjoint idempotents is dense in the set of all self-adjoint elements of $\mathcal{A}([2])$. It is clear that every von Neumann algebra is a $C^{*}$-algebra of real rank zero. In particular, $\mathcal{B}(H)$, the algebra of all bounded linear operators on a complex Hilbert space, has real rank zero. In this section, we assume that $\mathcal{A}$ is a von Neumann algebra acting on a complex Hilbert space $H$.

For a unital Banach algebra $\mathcal{B}, \mathcal{Z}(\mathcal{B})$ denotes the centre of $\mathcal{B}$, that is, $\mathcal{Z}(\mathcal{B})=$ 
$\{b \in \mathcal{B} \mid b x=x b$ for every $x \in \mathcal{B}\} . \mathcal{B}$ is called a factor if $\mathcal{Z}(\mathcal{B})=\mathbb{C} I$, where $I$ is the unit of $\mathcal{B}$ and $\mathbb{C}$ is the complex plane as usual. Two elements are orthogonal if they are commutative and their product is zero.

Theorem 2.1. Let $\mathcal{A}$ be a von Neumann algebra and $\mathcal{B}$ be a factor Banach algebra containing unit. Suppose that $\Phi: \mathcal{A} \rightarrow \mathcal{B}$ is a bounded surjective linear map. Then the following are equivalent.

(1) $\Phi$ preserves zero products.

(2) $\Phi$ is a homomorphism multiplied by a nonzero scalar.

Proof: It is clear that we need only to prove (1) implies (2). Assume that $\Phi$ : $\mathcal{A} \rightarrow \mathcal{B}$ is a bounded surjective linear map preserving zero products. First we shall show that $\Phi(I)=\lambda I$ for some nonzero $\lambda \in \mathbb{C}$.

Let $Q \in \mathcal{A}$ be an arbitrary idempotent, then by our assumptions, $Q(I-Q)=0$ implies that $\Phi(Q) \Phi(I-Q)=0$, so

$$
\Phi(Q) \Phi(I)=\Phi(I) \Phi(Q)=\Phi(Q)^{2} .
$$

It is well known that the subspace of all real linear combinations of projections (that is, self-adjoint idempotents) is dense under the norm topology in the subset of all selfadjoint elements of a von Neumann algebra. Since $\Phi$ is continuous, we get $\Phi(S) \Phi(I)=$ $\Phi(I) \Phi(S)$ for all $S \in \mathcal{A}$. Thus $\Phi(I) \in \mathcal{Z}(\mathcal{B})$ by the surjectivity of $\Phi$. Therefore $\Phi(I)=\lambda I$ for some $\lambda \in \mathbb{C}$ as $\mathcal{B}$ is a factor.

Next we prove that $\lambda \neq 0$. Otherwise, $\Phi(I)=0$, so, by equation $(2.1), \Phi(Q)^{2}=0$ for every idempotent $Q$ in $\mathcal{A}$. If $A=\sum_{i=1}^{n} \alpha_{i} P_{i}$, where $P_{i}(i=1, \ldots, n)$ are orthogonal projections and $\alpha_{i}$ are real numbers, then $\Phi(A)^{2}=\sum_{i=1}^{n} \alpha_{i}^{2} \Phi\left(P_{i}\right)^{2}=0$. It follows from the boundedness of $\Phi$ that, for every self-adjoint element $A$ in $\mathcal{A}$, we always have $\Phi(A)^{2}=0$. Now for arbitrary two self-adjoint elements $A$ and $B$ in $\mathcal{A}$, we have $0=\Phi(A+B)^{2}=\Phi(A) \Phi(B)+\Phi(B) \Phi(A)$. Let $T=T_{1}+i T_{2}$ be an arbitrary element in $\mathcal{A}$, where $T_{1}, T_{2}$ are the real and imaginary parts of $T$, respectively. Now it follows from the self-adjointness of $T_{1}$ and $T_{2}$ that $\Phi(T)^{2}=0$. Thus the range of $\Phi$ consists of square zero elements, which contradicts the surjectivity of $\Phi$ because the Banach algebra $\mathcal{B}$ contains elements other than square zero elements, for example, the unit $I$. Hence $\lambda \neq 0$. Without loss of generality, in the sequel, we may assume that $\lambda=1$, so $\Phi(I)=I$.

Our next step shall show that $\Phi$ preserves idempotent elements. Indeed, $Q$ is an idempotent if and only if $Q(I-Q)=0$. According to our assumptions, this implies that $\Phi(Q) \Phi(I-Q)=0$, so $\Phi(Q)=\Phi(Q)^{2}$ as $\Phi(I)=I$. That is, $\Phi(Q)$ is an idempotent.

Now let us prove that $\Phi$ is a Jordan homomorphism. Because $\Phi$ preserves idempotent elements, it maps a set of orthogonal idempotents of $\mathcal{A}$ into a set of orthogonal 
idempotents of $\mathcal{B}$. If $A=A^{*}$ is a self-adjoint element in the von Neumann algebra $\mathcal{A}$, then $A$ is a limit of a sequence of real linear combinations of orthogonal projections and consequently, by the continuity of $\Phi, \Phi(A)$ is a limit of the sequence of real linear combinations of orthogonal idempotents. It follows that $\Phi\left(A^{2}\right)=\Phi(A)^{2}$ holds for every self-adjoint element $A$. Replacing $A$ by $C+D$ in this relation, where $C$ and $D$ are self-adjoint, we get $\Phi(C D+D C)=\Phi(C) \Phi(D)+\Phi(D) \Phi(C)$. For any $T \in \mathcal{A}$, write $T=T_{1}+i T_{2}$, where $T_{1}, T_{2}$ are real and imaginary parts of $T$, respectively. Then the self-adjointness of $T_{1}$ and $T_{2}$ yields that

$$
\begin{aligned}
\Phi\left(T^{2}\right) & =\Phi\left(T_{1}^{2}-T_{2}^{2}+i\left(T_{1} T_{2}+T_{2} T_{1}\right)\right) \\
& =\Phi\left(T_{1}\right)^{2}-\Phi\left(T_{2}\right)^{2}+i\left(\Phi\left(T_{1}\right) \Phi\left(T_{2}\right)+\Phi\left(T_{2}\right) \Phi\left(T_{1}\right)\right)=\Phi(T)^{2}
\end{aligned}
$$

Hence $\Phi$ is a Jordan homomorphism.

Finally, we prove that $\Phi$ is a homomorphism. Let $A, B \in \mathcal{A}$ be such that $A B=0$, then $\Phi(A) \Phi(B)=0$ and

$$
\Phi(B A)=\Phi(B A+A B)=\Phi(B) \Phi(A)+\Phi(A) \Phi(B)=\Phi(B) \Phi(A) .
$$

For any idempotent $P \in \mathcal{A}$ and arbitrary $T \in \mathcal{A}$, since $T P(I-P)=0$, we have $\Phi(T P)=\Phi(T P) \Phi(P)$. It follows that

$$
\begin{aligned}
\Phi(P T)-\Phi(P T P) & =\Phi(P T(I-P))=\Phi(P) \Phi(T(I-P)) \\
& =\Phi(P) \Phi(T)-\Phi(P) \Phi(T P) \\
& =\Phi(P) \Phi(T)-\Phi(P) \Phi(T P) \Phi(P) \\
& =\Phi(P) \Phi(T)-\Phi(P T P),
\end{aligned}
$$

hence we have $\Phi(P T)=\Phi(P) \Phi(T)$. Now it is clear that $\Phi(S T)=\Phi(S) \Phi(T)$ for any $S$ and $T$ in $\mathcal{A}$, so $\Phi$ is a homomorphism.

Corollary 2.2. Let $\mathcal{A}$ be a von Neumann algebra and $\mathcal{B}$ be a factor Banach algebra. Suppose that $\Phi: \mathcal{A} \rightarrow \mathcal{B}$ is a bounded linear map. Then the following are equivalent.

(1) $\Phi$ is a surjection preserving zero products in both directions.

(2) $\Phi$ is a bijection preserving zero products.

(3) $\Phi$ is an isomorphism multiplied by a nonzero scalar.

ProOF: (3) implies (1) is clear. As to (1) implies (2), we need only to prove that $\Phi$ is injective. Assume that $\Phi(T)=0$. For any $S \in \mathcal{A}$, we have $\Phi(T) \Phi(S)=0$ and hence $T S=0$ as $\Phi$ preserves the zero products in both directions. So $T=0$. That is, $\Phi$ is injective. 
That (2) implies (3) follows from Theorem 2.1.

In Theorem 2.1, when the range of $\Phi$ is a von Neumann algebra, we have more general result. Let $\mathcal{B}$ be a von Neumann algebra acting on complex Hilbert space $K$ and let $E . F \in \mathcal{B}$ be two projections. We say that $E$ and $F$ are equivalent, written as $E \sim F$, if there exists a partial isometry $V \in \mathcal{B}$ such that $E=V^{*} V$ and $F=V V^{*}$. The relation $E \preccurlyeq F$ means that $E$ is equivalent to a subprojection of $F$. Assume that $\left\{P_{\lambda} \mid \lambda \in \Lambda\right\}$ in $\mathcal{Z}(B)$ is an orthogonal projection family satisfying $\sum_{\lambda \in \Lambda} P_{\lambda}=I$. If for any orthogonal family $\left\{Q_{\alpha} \mid \alpha \in \Delta\right\}$ of projections in $\mathcal{Z}(\mathcal{B})$ with $\sum_{\alpha \in \Delta}^{\lambda \in \Lambda} Q_{\alpha}=I$ and if for any $\alpha \in \Delta$, there exists $\lambda \in \Lambda$ such that $P_{\lambda} \preccurlyeq Q_{\alpha}$, then we say that $\left\{P_{\lambda} \mid \lambda \in \Lambda\right\}$ is a maximal orthogonal projection family in $\mathcal{Z}(\mathcal{B})$. In the following results we assume that $\mathcal{B}$ has a maximal orthogonal projection family in $\mathcal{Z}(\mathcal{B})$.

TheOREM 2.3. Let $\mathcal{A}$ and $\mathcal{B}$ be von Neumann algebras acting on complex Hilbert spaces $H$ and $K$, respectively. Suppose that $\Phi: \mathcal{A} \rightarrow \mathcal{B}$ is a bounded surjective linear map, then the following are equivalent.

(1) $\Phi$ preserves zero products.

(2) There exists an invertible element $D \in \mathcal{Z}(\mathcal{B})$ and a homomorphism $\Psi$ : $\mathcal{A} \rightarrow \mathcal{B}$ such that $\Phi(T)=D \Psi(T)$ for all $T \in \mathcal{A}$.

Proof: As (2) implies (1) is obvious, we need only to prove (1) implies (2). Assume (1). From the proof of Theorem 2.1, we have $\Phi(I)=D \in \mathcal{Z}(\mathcal{B})$. Take a maximal orthogonal projection family $\left\{P_{\lambda} \mid \lambda \in \Lambda\right\}$ in $\mathcal{Z}(\mathcal{B})$ so that $\sum_{\lambda \in \Lambda} P_{\lambda}=I$. Let $K_{\lambda}=P_{\lambda} K$, then $K=\sum_{\lambda \in \Lambda}^{\oplus} K_{\lambda}$. For any $S \in \mathcal{B}$, let $S_{\lambda}=\left.S\right|_{K_{\lambda}}$, we have $S=\sum_{\lambda \in \Lambda}^{\oplus} S_{\lambda}$. Put $\mathcal{B}_{\lambda}=\left\{S_{\lambda} \mid S \in \mathcal{B}\right\} \subset \mathcal{B}\left(K_{\lambda}\right)$, then it is clear that $\mathcal{B}_{\lambda}$ is a factor von Neumann algebra on $K_{\lambda}$ and $\mathcal{B}=\sum_{\lambda \in \Lambda}^{\oplus} \mathcal{B}_{\lambda}$. Otherwise, assume that there exist $\lambda_{0} \in \Lambda$ such that $\mathcal{B}_{\lambda_{0}}$ is not a factor von Neumann algebra on $K_{\lambda_{0}}$. Then there exist nonzero subprojections $P_{\alpha_{1}}$ and $P_{\alpha_{2}} \in \mathcal{Z}(\mathcal{B})$ of $P_{\lambda_{0}}$ such that $P_{\alpha_{1}}+P_{\alpha_{2}}=P_{\lambda_{0}}$. So $\left\{P_{\alpha_{1}}, P_{\alpha_{2}}, P_{\lambda} \mid \lambda \in \Lambda\right.$ and $\left.\lambda \neq \lambda_{0}\right\}$ is an orthogonal projection family and $P_{\alpha_{1}}+P_{\alpha_{2}}+\sum_{\lambda \in \Lambda, \lambda \neq \lambda_{0}} P_{\lambda}=I$, which contradicts the maximality of $\left\{P_{\lambda} \mid \lambda \in \Lambda\right\}$. Hence $D=\sum_{\lambda \in \Lambda}^{\oplus} \alpha(\lambda) I_{\lambda}$, where, for each $\lambda \in \Lambda, I_{\lambda}$ is the identity operator on Hilbert space $K_{\lambda}$ and $\alpha(\lambda)$ is a scalar.

Now, for every $\lambda \in \Lambda$, define $\Phi_{\lambda}(T)=\left.\Phi(T)\right|_{K_{\lambda}}$ for every $T \in \mathcal{A}$. Then $\Phi_{\lambda}: \mathcal{A} \rightarrow$ $\mathcal{B}_{\lambda}$ is linear and is zero product preserving. It is clear that $\Phi(\cdot)=\sum_{\lambda \in \Lambda}^{\oplus} \Phi_{\lambda}(\cdot)$. Since $\Phi$ is surjective, $\Phi_{\lambda}$ is also surjective. By Theorem 2.1, we have that $\Phi_{\lambda}(I)=\alpha(\lambda) I_{\lambda} \neq 0$ and there exists a homomorphism $\Psi_{\lambda}: \mathcal{A} \rightarrow \mathcal{B}_{\lambda}$ such that $\Phi_{\lambda}=\alpha(\lambda) \Psi_{\lambda}$. Let $\Psi=\sum_{\lambda \in \Lambda}^{\oplus} \Psi_{\lambda}$, 
then $\Psi: \mathcal{A} \rightarrow \mathcal{B}$ is a homomorphism and $\Phi(\cdot)=D \Psi(\cdot)$. We claim that the normal element $D$ is invertible, since, otherwise, no element in the range of $\Phi$ can be invertible, which contradicts the surjectivity of $\Phi$. This completes the proof.

Corollary 2.4. Let $\mathcal{A}$ and $\mathcal{B}$ be two von Neumann algebras acting on a complex Hilbert spaces $H$ and $K$, respectively. Suppose that $\Phi: \mathcal{A} \rightarrow \mathcal{B}$ is a bounded linear map. Then the following are equivalent.

(1) $\Phi$ is a surjection preserving zero products in both directions.

(2) $\Phi$ is a bijection preserving zero products.

(3) There exists an invertible element $D \in \mathcal{Z}(\mathcal{B})$ and an isomorphism $\Psi$ : $\mathcal{A} \rightarrow \mathcal{B}$ such that $\Phi(T)=D \Psi(T)$ for every $T \in \mathcal{A}$.

Proof: This is immediate from Corollary 2.2 and Theorem 2.3.

\section{LiNEAR MAPS PRESERVING TR-RANK}

In this section, we discuss the linear maps on finite von Neumann algebras which are completely tr-rank nonincreasing and give a characterisation of spatial *automorphisms. Before defining the conception of tr-rank for an element in finite von Neumann algebras, we first recall some notions. Let $\mathcal{R}$ be a von Neumann algebra acting on Hilbert space $H$. A projection $E$ in a von Neumann algebra $\mathcal{R}$ is said to be finite relative to $\mathcal{R}$ if there is no proper subprojection of $E$ that is equivalent to $E$. We say that $\mathcal{R}$ is finite if the identity $I \in \mathcal{R}$ is a finite projection relative to $\mathcal{R}$. For any positive integer $n$, let $\mathcal{R} \otimes M_{n}(\mathbb{C})=\left\{\mathbf{T}=\left(T_{i j}\right)_{n \times n} \mid T_{i j} \in \mathcal{R}\right\}$, which is still a finite von Neumann algebra in $\mathcal{B}\left(H^{(n)}\right)$, where $H^{(n)}$ is the direct sum of $n$ copies of $H$. It is well known that a von Neumann algebra is finite if and only if it has a faithful normal centre-valued trace (see [10]).

Definition 3.1: Let $\mathcal{R}$ be a finite von Neumann algebra on a Hilbert space $H$ and $\rho$ be its unique centre-valued trace satisfying $\rho(A)=A$ whenever $A$ is in the centre. For any $\mathbf{T}={ }_{f}\left(T_{i j}\right)_{n \times n} \in \mathcal{R} \otimes M_{n}(\mathbb{C})$, define $\rho_{n}(\mathbf{T})=\sum_{i=1}^{n} \rho\left(T_{i i}\right)$ and $\operatorname{tr}-\operatorname{rank}(\mathbf{A})=\rho_{n}\left(R_{\mathbf{T}}\right)$, where $R_{\mathbf{T}}$ stands for the projection onto the closure of the range of $\mathbf{T}$ as an operator on $H^{(n)}$.

Note that $\operatorname{tr}-\operatorname{rank}(\mathbf{T})=0$ implies $\mathbf{T}=0$ since the trace $\rho$ is faithful.

Let $\mathcal{M}$ and $\mathcal{N}$ be two finite von Neumann algebras, the linear map $\Phi: \mathcal{M} \rightarrow \mathcal{N}$ is called completely tr-rank nonincreasing (or, preserving) if $\Phi_{n}: \mathcal{M} \otimes M_{n}(\mathbb{C}) \rightarrow$ $\mathcal{N} \otimes M_{n}(\mathbb{C})$ defined by $\Phi_{n}\left(\left(T_{i j}\right)_{n \times n}\right)=\left(\Phi\left(T_{i j}\right)\right)_{n \times n}$ is tr-rank nonincreasing (or, preserving) for every natural number $n$.

Before proving the main result of this section, we investigate some properties relative to tr-rank, which are useful in our study. It is seen from the following proposition 
that tr-rank is a suitable generalisation of the rank of matrices over $\mathbb{C}$ to that of the matrices over finite von Neumann algebras.

For an operator $T$, we denote its kernel and range by $\operatorname{ker}(T)$ and $\operatorname{ran}(T)$, respectively.

PRoposition 3.2. Let $\mathcal{R}$ be a finite von Neumann algebra acting on Hilbert space $H$. Let $\mathbf{A}, \mathbf{B}, \mathbf{E}$ and $\mathbf{F} \in \mathcal{R} \otimes M_{n}(\mathbb{C})$ with $\mathbf{E}$ and $\mathbf{F}$ being projections. Then the following statements are true.

(1) $\mathbf{E} \sim \mathbf{F}$ if and only if $\operatorname{tr}-\operatorname{rank}(\mathbf{E})=\operatorname{tr}-\operatorname{rank}(\mathbf{F})$;

(2) $\mathbf{E} \preccurlyeq \mathbf{F}$ if and only if $\operatorname{tr}-\operatorname{rank}(\mathbf{E}) \leqslant \operatorname{tr}-\operatorname{rank}(\mathbf{F})$;

(3) if $\mathbf{E A}=\mathbf{A}$, then $\operatorname{tr}-\operatorname{rank}(\mathbf{A}) \leqslant \operatorname{tr}-\operatorname{rank}(\mathbf{E})$;

(4) $\operatorname{tr}-\operatorname{rank}(\mathbf{A B}) \leqslant \min \{\operatorname{tr}-\operatorname{rank}(\mathbf{A}), \operatorname{tr}-\operatorname{rank}(\mathbf{B})\}$;

(5) if $\mathbf{B}$ is invertible, then $\operatorname{tr}-\operatorname{rank}(\mathbf{B A})=\operatorname{tr}-\operatorname{rank}(\mathbf{A B})=\operatorname{tr}-\operatorname{rank}(\mathbf{A})$;

(6) if $\mathbf{T}=\left(T_{i j}\right)_{n \times n} \in \mathcal{R} \otimes M_{n}(\mathbb{C})$ satisfies $T_{i j}=A$ for all $(i, j)$, then

$$
\operatorname{tr}-\operatorname{rank}(\mathbf{T})=\operatorname{tr}-\operatorname{rank}(A) .
$$

Proof: (1) By the Definition 3.1, it is easily checked that $\rho_{n}(\mathbf{A B})=\rho_{n}(\mathbf{B A})$ for any $\mathbf{A}, \mathbf{B} \in \mathcal{R} \otimes M_{n}(\mathbb{C})$. If $\mathbf{E}$ and $\mathbf{F}$ are projections such that $\mathbf{E} \sim \mathbf{F}$, then there exists a partial isometry $\mathrm{V} \in \mathcal{R} \otimes M_{n}(\mathbb{C})$ such that $\mathbf{E}=\mathrm{V}^{*} \mathrm{~V}$ and $\mathbf{F}=\mathrm{V} V^{*}$. Thus

$$
\operatorname{tr}-\operatorname{rank}(\mathbf{E})=\rho_{n}(\mathbf{E})=\rho_{n}\left(\mathbf{V}^{*} \mathbf{V}\right)=\rho_{n}\left(\mathbf{V} \mathbf{V}^{*}\right)=\rho_{n}(\mathbf{F})=\operatorname{tr}-\operatorname{rank}(\mathbf{F})
$$

(2) $\mathbf{E} \preccurlyeq \mathbf{F}$ if and only if there exists a subprojection $\mathbf{F}_{1} \leqslant \mathbf{F}$ such that $\mathbf{E} \sim \mathbf{F}_{1}$. By the positivity of $\rho_{n}$ and (1), we see that (2) is true.

(3) Let $\mathbf{A}=\mathbf{V}|\mathbf{A}|$ be the polar decomposition of $\mathbf{A}$. Since $\mathbf{E A}=\mathbf{A}$ and $\operatorname{ker} \mathbf{A}=\operatorname{ker}(|\mathbf{A}|)=\operatorname{ker} \mathbf{V}$, for any $x \in \operatorname{ker} \mathbf{A}$, we have $(\mathbf{I}-\mathbf{E}) \mathbf{V} x=0$ and therefore, $\left.(\mathbf{I}-\mathbf{E}) \mathbf{V}\right|_{\text {ker } \mathbf{A}}=0$; for any $y \in \operatorname{ran}(|\mathbf{A}|)$, there exists $x \in(\operatorname{ker}|\mathbf{A}|)^{\perp}=(\operatorname{ker} \mathbf{A})^{\perp}$ such that $y=|\mathbf{A}| x$, hence $(\mathbf{I}-\mathbf{E}) \mathbf{V} y=(\mathbf{I}-\mathbf{E}) \mathbf{V}|\mathbf{A}| x=0$ and consequently, $\left.(\mathbf{I}-\mathbf{E}) \mathbf{V}\right|_{(\operatorname{ker} \mathbf{A})^{\perp}}=0$ since $(\operatorname{ker} \mathbf{A})^{\perp}=\overline{\operatorname{ran}(|\mathbf{A}|)}$. So $(\mathbf{I}-\mathbf{E}) \mathbf{V}=0$ and $(\mathbf{I}-\mathbf{E}) \mathbf{V} V^{*}=$ 0 , which implies that $R_{\mathbf{A}}=\mathbf{V V}^{*} \leqslant \mathbf{E}$. By property $(2), \operatorname{tr}-\operatorname{rank}(\mathbf{A}) \leqslant \operatorname{tr}-\operatorname{rank}(\mathbf{E})$.

(4) Since $R_{\mathbf{A}} \mathrm{AB}=\mathbf{A} B, \mathrm{AB}=\mathbf{A} B R_{\mathrm{B}^{*}}$ and $R_{\mathrm{B}} \sim R_{\mathbf{B}^{*}}$, (4) follows from (1) and (3).

(5) By (4) and tr-rank (B) $=n I$ if $\mathrm{B}$ is invertible, we get

$$
\operatorname{tr}-\operatorname{rank}(\mathbf{A B}) \leqslant \min \{\operatorname{tr}-\operatorname{rank}(\mathbf{A}), n I\}=\operatorname{tr}-\operatorname{rank}(\mathbf{A})
$$

and

$$
\operatorname{tr}-\operatorname{rank}(\mathbf{A})=\operatorname{tr}-\operatorname{rank}\left(\mathbf{A B B}^{-1}\right) \leqslant \min \{\operatorname{tr}-\operatorname{rank}(\mathbf{A B}), n I\}=\operatorname{tr}-\operatorname{rank}(\mathbf{A B}) .
$$


So (5) is true.

(6) Let

$$
\mathbf{W}=\left(\begin{array}{cccccc}
I & -I & 0 & \cdots & 0 & 0 \\
0 & I & -I & \cdots & 0 & 0 \\
0 & 0 & I & \cdots & 0 & 0 \\
\vdots & \vdots & \vdots & \ddots & \vdots & \vdots \\
0 & 0 & 0 & \cdots & I & -I \\
0 & 0 & 0 & \cdots & 0 & I
\end{array}\right)
$$

and

$$
\mathbf{V}=\left(\begin{array}{cccccc}
I & 0 & 0 & \cdots & 0 & 0 \\
-I & I & 0 & \cdots & 0 & 0 \\
0 & -I & I & \cdots & 0 & 0 \\
\vdots & \vdots & \vdots & \ddots & \vdots & \vdots \\
0 & 0 & 0 & \cdots & I & 0 \\
0 & 0 & 0 & \cdots & -I & I
\end{array}\right) .
$$

Then $\mathbf{W}$ and $\mathbf{V}$ are invertible and

$$
\mathbf{W T V}=\left(\begin{array}{cccccc}
0 & 0 & 0 & \cdots & 0 & 0 \\
0 & 0 & 0 & \cdots & 0 & 0 \\
0 & 0 & 0 & \cdots & 0 & 0 \\
\vdots & \vdots & \vdots & \ddots & \vdots & \vdots \\
0 & 0 & 0 & \cdots & 0 & 0 \\
0 & 0 & 0 & \cdots & 0 & A
\end{array}\right)
$$

Hence, by (5), we get $\operatorname{tr}-\operatorname{rank}(\mathbf{T})=\operatorname{tr}-\operatorname{rank}(\mathbf{W T V})=\operatorname{tr}-\operatorname{rank}(A)$.

We are now in a position to prove the main theorem in this section.

TheOREM 3.3. Let $\mathcal{R}$ be a finite von Neumann algebra and $\mathcal{M}$ be a linear subspace of $\mathcal{R}$ containing the unit I. Suppose that $\Phi: \mathcal{M} \rightarrow \mathcal{R}$ is a completely tr-rank nonincreasing (or, preserving) unital (that is, $\Phi(I)=I$ ) linear map. Then $\Phi$ can be extended to a completely tr-rank nonincreasing (or, preserving) algebraic homomorphism from the subalgebra of $\mathcal{R}$ generated by $\mathcal{M}$ into $\mathcal{R}$. 
Proof: For any $T_{i} \in \mathcal{M}(i=1, \ldots, k)$, let

$$
\begin{aligned}
& \mathbf{T}=\left(\begin{array}{cccccc}
T_{1} & -I & 0 & \cdots & 0 & 0 \\
0 & T_{2} & -I & \cdots & 0 & 0 \\
0 & 0 & T_{3} & \cdots & 0 & 0 \\
\vdots & \vdots & \vdots & \ddots & \vdots & \vdots \\
0 & 0 & 0 & \cdots & T_{k-1} & -I \\
0 & 0 & 0 & \cdots & 0 & T_{k}
\end{array}\right) \\
& \mathbf{A}=\left(\begin{array}{cccccc}
-I & 0 & 0 & \cdots & 0 & 0 \\
-T_{2} & -I & 0 & \cdots & 0 & 0 \\
-T_{3} T_{2} & -T_{3} & -I & \cdots & 0 & 0 \\
\vdots & \vdots & \vdots & \ddots & \vdots & \vdots \\
-T_{k-1} \cdots T_{2} & -T_{k-1} \cdots T_{3} & -T_{k-1} \cdots T_{4} & \cdots & -I & 0 \\
T_{k} \cdots T_{2} & T_{k} \cdots T_{3} & T_{k} \cdots T_{4} & \cdots & T_{k} & I
\end{array}\right)
\end{aligned}
$$

and

$$
\mathbf{B}=\left(\begin{array}{cccccc}
0 & 0 & 0 & \cdots & 0 & I \\
I & 0 & 0 & \cdots & 0 & T_{1} \\
0 & I & 0 & \cdots & 0 & T_{2} T_{1} \\
\vdots & \vdots & \vdots & \ddots & \vdots & \vdots \\
0 & 0 & 0 & \cdots & 0 & T_{k-2} \cdots T_{1} \\
0 & 0 & 0 & \cdots & I & T_{k-1} \cdots T_{1}
\end{array}\right)
$$

Then $\mathbf{A}, \mathbf{T}$, and $\mathbf{B} \in \mathcal{M} \otimes M_{k}(\mathbb{C})$ and a straightforward computation shows that

$$
\mathbf{A T B}=\left(\begin{array}{cccccc}
I & 0 & 0 & \cdots & 0 & 0 \\
0 & I & 0 & \cdots & 0 & 0 \\
0 & 0 & I & \cdots & 0 & 0 \\
\vdots & \vdots & \vdots & \ddots & \vdots & \vdots \\
0 & 0 & 0 & \cdots & I & 0 \\
0 & 0 & 0 & \cdots & 0 & T_{k} \cdots T_{1}
\end{array}\right) .
$$

It is clear that both $\mathbf{A}$ and $\mathbf{B}$ are invertible, so $\operatorname{tr}-\operatorname{rank}(\mathbf{T})=\operatorname{tr}-\operatorname{rank}(\mathbf{A T B})$ by property (5) of Proposition 3.2 and

$$
(k-1) I+\operatorname{tr}-\operatorname{rank}\left(T_{k} \cdots T_{2} T_{1}\right)=\operatorname{tr}-\operatorname{rank}(\mathbf{T}) .
$$

Hence we have

$$
\begin{aligned}
(k-1) I+\operatorname{tr}-\operatorname{rank}\left(\Phi\left(T_{k}\right) \cdots \Phi\left(T_{2}\right) \Phi\left(T_{1}\right)\right) & =\operatorname{tr}-\operatorname{rank}\left(\Phi_{k}(\mathrm{~T})\right) \leqslant \operatorname{tr}-\operatorname{rank}(\mathrm{T}) \\
& =(k-1) I+\operatorname{tr}-\operatorname{rank}\left(T_{k} \cdots T_{2} T_{1}\right)
\end{aligned}
$$


because $\Phi$ is unital and completely tr-rank nonincreasing. Now it is clear that

$$
\operatorname{tr}-\operatorname{rank}\left(\Phi\left(T_{k}\right) \cdots \Phi\left(T_{2}\right) \Phi\left(T_{1}\right)\right) \leqslant \operatorname{tr}-\operatorname{rank}\left(T_{k} \cdots T_{2} T_{1}\right)
$$

namely, the tr-rank of the product of images of a unital completely tr-rank nonincreasing linear map at some elements is not larger than the tr-rank of the corresponding product of these elements.

Now assume that $T \in \mathcal{R}$ has the form

$$
T=\sum_{r=1}^{m} S_{r 1} \cdots S_{r k_{r}} \text { where } S_{r s_{r}} \in \mathcal{M} \text { for all } r=1, \ldots, m \text { and } s_{r}=1, \ldots, k_{r}
$$

Let $\mathbf{W}_{r s_{r}}=\left(W_{i j}^{\left(r s_{r}\right)}\right)_{m \times m} \in \mathcal{M} \otimes M_{m}(\mathbb{C})$ with $W_{r r}^{\left(r s_{r}\right)}=S_{r s_{r}}, W_{i i}^{\left(r s_{r}\right)}=I$ if $i \neq r$ and $W_{i j}^{\left(r s_{r}\right)}=0$ if $i \neq j ; r=1, \ldots, m$ and $s_{r}=1, \ldots, k_{r}$. Let $\mathbf{A}=\left(A_{i j}\right)_{m \times m}$ with $A_{i j}=I$ for all $(i, j)$. Then

$$
\mathbf{A W}_{11} \cdots \mathbf{W}_{1 k_{1}} \mathbf{W}_{21} \cdots \mathbf{W}_{(m-1) k_{(m-1)}} \mathbf{W}_{m \mathbf{1}} \cdots \mathbf{W}_{m k_{m}} \mathbf{A}=\mathbf{V}=\left(V_{i j}\right)_{m \times m}
$$

with $V_{i j}=T=\sum_{r=1}^{m} S_{r 1} \cdots S_{r k_{r}}$ for every $i, j=1, \ldots, m$. It follows from Proposition $3.2(6)$ that

$$
\operatorname{tr}-\operatorname{rank}\left(\sum_{r=1}^{m} S_{r 1} \cdots S_{r k_{r}}\right)=\operatorname{tr}-\operatorname{rank}(\mathrm{V})
$$

Let

$$
\begin{aligned}
\mathbf{B}=\left(B_{i j}\right)=\Phi_{m}(\mathbf{A}) \Phi_{m}\left(\mathbf{W}_{11}\right) & \cdots \Phi_{m}\left(\mathbf{W}_{1 k_{1}}\right) \Phi_{m}\left(\mathbf{W}_{21}\right) \\
& \cdots \Phi_{m}\left(\mathbf{W}_{(m-1) k_{(m-1)}}\right) \Phi_{m}\left(\mathbf{W}_{m 1}\right) \cdots \Phi_{m}\left(\mathbf{W}_{m k_{m}}\right) \Phi_{m}(\mathbf{A})
\end{aligned}
$$

then $B_{i j}=S=\sum_{r=1}^{m} \Phi\left(S_{r 1}\right) \cdots \Phi\left(S_{r k_{r}}\right)$ for every $i, j=1, \ldots, m$. Similarly,

$$
\operatorname{tr}-\operatorname{rank}(\mathbf{B})=\operatorname{tr}-\operatorname{rank}\left(\sum_{r=1}^{m} \Phi\left(S_{r 1}\right) \cdots \Phi\left(S_{r k_{r}}\right)\right)
$$

Now, since $\Phi_{m}: \mathcal{M} \otimes M_{m}(\mathbb{C}) \rightarrow \mathcal{R} \otimes M_{m}(\mathbb{C})$ is completely tr-rank nonincreasing, similar to inequality $(3.1)$ we have

$$
\operatorname{tr}-\operatorname{rank}(B) \leqslant \operatorname{tr}-\operatorname{rank}(\mathbf{V})
$$


So it follows that

$$
\operatorname{tr}-\operatorname{rank}\left(\sum_{r=1}^{m} \Phi\left(S_{r 1}\right) \cdots \Phi\left(S_{r k_{r}}\right)\right) \leqslant \operatorname{tr}-\operatorname{rank}\left(\sum_{r=1}^{m} S_{r 1} \cdots S_{r k_{r}}\right) .
$$

Let $\widehat{\mathcal{M}}$ be the subalgebra of $\mathcal{R}$ generated by $\mathcal{M}$ and let $\widehat{\Phi}: \widehat{\mathcal{M}} \rightarrow \mathcal{R}$ be a linear map determined by

$$
\widehat{\Phi}\left(\sum_{r=1}^{m} S_{r 1} \cdots S_{r k_{r}}\right)=\left(\sum_{r=1}^{m} \Phi\left(S_{r 1}\right) \cdots \Phi\left(S_{r k_{r}}\right)\right) .
$$

If $\sum_{r=1}^{m} S_{r 1} \cdots S_{r k_{r}}=0$, then inequality (3.2) forces $\sum_{r=1}^{m} \Phi\left(S_{r 1}\right) \cdots \Phi\left(S_{r k_{r}}\right)=0$. In particular, it follows from this fact that, if $T, S$ and $T S \in \mathcal{M}$, then $\Phi(T S)=\Phi(T) \Phi(S)$. Thus $\widehat{\Phi}$ is well defined as an extension of $\Phi$ and is an algebraic homomorphism. Now $\widehat{\Phi}$ is also completely tr-rank nonincreasing since $\widehat{\Phi}_{m}=\widehat{\Phi_{m}}$.

If $\Phi$ is completely tr-rank preserving, then the inequalities between "tr-rank" in the above argument are exact equalities, so $\widehat{\Phi}$ is completely tr-rank preserving in this case. In particular, $\widehat{\Phi}$ is injective.

Obviously, when $\mathcal{M}$ is a subalgebra of $\mathcal{R}$, we have the following corollary.

Corollary 3.4. Let $\mathcal{R}$ be a finite von Neumann algebra. Suppose that $\mathcal{M}$ is a subalgebra of $\mathcal{R}$ and $\Phi: \mathcal{M} \rightarrow \mathcal{R}$ is a completely tr-rank nonincreasing (or, preserving) unital linear map, then $\Phi$ is an algebraic homomorphism (or, injective homomorphism).

The following lemma can be found in [6, Corollary, p. 305], but for convenience we state it here.

Lemma 3.5. Suppose that $\mathcal{R}$ is a finite von Neumann algebra and $\mathcal{Z}$ is its centre. Let $\Phi$ be an ${ }^{*}$-automorphism of $\mathcal{R}$ which leaves the elements of $\mathcal{Z}$ fixed. Then $\Phi$ is spatial.

Recall that a map $\Psi$ from a von Neumann algebra $\mathcal{R}$ into itself is self-adjoint if it preserves the involution operation, that is, it satisfies the condition $\Psi\left(T^{*}\right)=\Psi(T)^{*}$ for all $T$ in $\mathcal{R} ; \Psi$ is called a ${ }^{*}$-automorphism of $\mathcal{R}$ if it is self-adjoint and algebraically automorphic. A *automorphism $\Psi$ of a von Neumann algebra $\mathcal{R}$ acting on a Hilbert space $H$ is said to be spatial if there exists a unitary operator $U \in \mathcal{B}(H)$ such that $\Psi(T)=U T U^{*}$ for all $T$ in $\mathcal{R}$. Also recall that the central carrier $C_{T}$ of an operator $T$ in $\mathcal{R}$ is the projection $I-P$, where $P$ is the union of all central projections $P_{\alpha}$ in $\mathcal{R}$ such that $P_{\alpha} T=0$. That is, $C_{T}$ is the smallest projection $Q$ in the centre $\mathcal{Z}=\mathcal{R} \cap \mathcal{R}^{\prime}$ of $\mathcal{R}$ for which $Q T=T$. 
THEOREM 3.6. Let $\mathcal{R}$ be a finite von Neumann algebra and let $\Phi: \mathcal{R} \rightarrow \mathcal{R}$ be a unital self-adjoint and surjective linear map. Then $\Phi$ is completely tr-rank preserving if and only if $\Phi$ is a spatial *automorphism of $\mathcal{R}$ that leaves the central elements fixed.

PROOF: The "if" part is obvious. To check the "only if" part, suppose that $\Phi$ is completely tr-rank preserving. By Corollary $3.4, \Phi$ is an automorphism of $\mathcal{R}$. It is obvious that, for any operator $A \in \mathcal{Z}$, we have $\Phi(A) \in \mathcal{Z}$ and $\left.\Phi\right|_{\mathcal{Z}}: \mathcal{Z} \rightarrow \mathcal{Z}$ is oneto-one and onto. Apparently, the map $\Phi$ is bounded and, for any projection $P \in \mathcal{Z}$, $\Phi(P) \in \mathcal{Z}$ is a projection since $\Phi$ is self-adjoint. Now the tr-rank preservativity of $\Phi$ implies that $\Phi(P)=P$ for every projection $P \in \mathcal{Z}$. Because every operator in $\mathcal{Z}$ is a norm limit of linear combinations of the orthogonal projections in $\mathcal{Z}$, it follows that $\Phi(Z)=Z$ for every $Z \in \mathcal{Z}$. By Lemma $3.5, \Phi$ is spatial.

\section{REFERENCES}

[1] M. Bresar and P. Semrl, 'Linear preservers on $\mathcal{B}(X)$ ', in Linear operators, Banach Center Publication 38 (Institute of Mathematics Polish Academy of Sciences, Warzawa, 1997), pp. $49-58$.

[2] L.G. Brown and G.K. Pedersen, ' $C$ *-algebras of real rank zero', J. Funct. Anal. 99 (1991), 131-149.

[3] W. Chooi and M. Lim, 'Linear preservers on triangular matrices', Linear Algebra Appl. 269 (1998), 241-255.

[4] J. Cui, J. Hou and B. Li, 'Linear preservers on upper triangular operator matrix algebras', Linear Algebra Appl. 336 (2001), 29-50.

[5] J. Cui and J. Hou, 'Characterizations of nest algebra automorphisms', Chinese Ann. Math. (to appear).

[6] J. Dixmier, Von Neumann algebras, North Holland Mathematical Library 27 (North Holland Publishing Company, Amsterdam, New York, Oxford, 1981).

[7] J. Hou, 'Rank preserving linear maps on $B(X)$ ', Sci. China Ser. A 32 (1989), 929-940.

[8] J. Hou, 'Multiplicative maps on $\mathcal{B}(X)$ ', Sci. China Ser. A 41 (1998), 337-345.

[9] J. Hou and M. Gao, 'Additive mappings on $\mathcal{B}(H)$ that preserves zero products', Kexue Tongbao (Chinese) 43 (1998), 2388-2392.

[10] R. Kadison and J. Ringrose, Fundamentals of the theory of operator algebras, Graduate Studies in Mathematics 16, Volume II (Academic Press, Inc., London, 1986).

[11] C.K. Li and N.K. Tsing, 'Linear preserver problems: a brief introduction and some special techniques', Linear Algebra Appl. 162/164 (1992), 217-235.

[12] M. Lim, 'Rank and tensor rank preservers', Linear and Multilinear Algebra 33 (1992), 7-21.

[13] L. Molnar, 'Some linear preserver problems on $\mathcal{B}(H)$ concerning rank and corank', Linear Algebra Appl. 286 (1999), 311-321.

[14] L. Molnar and P. Semrl, 'Some linear preserver problems on upper triangular matrices', Linear and Multilinear Algebra 45 (1998), 189-206. 
[15] P. Semrl, 'Linear mappings preserving square-zero matrices', Bull. Austral. Math. Soc. 48 (1993), 365-370.

[16] S. Wei and S. Hou, 'Rank preserving linear maps on nest algebra', J. Operator Theory 39 (1998), 207-217.

Institute of Mathematics

Chinese Academy of Sciences

Beijing 100080

Peoples Republic of China

Department of Mathematics

Shanxi Teachers University

Linfen 041004

Peoples Republic of China
Current address:

Department of Applied Mathematics

Taiyuan University of Technology

Taiyuan 030024

Peoples Republic of China

and

Department of Mathematics

Shanxi Teachers University

Linfen 041004

Peoples Republic of China

e-mail: cuijl@dns.sxtu.edu.cn

Current address:

Department of Mathematics

Shanxi University

Taiyuan 030000

Peoples Republic of China

e-mail: jhou@dns.sxtu.edu.cn 